\title{
Das Konzept der liebevolle Erzähler in Olga Tokarczuks Vorlesung zur Verleihung des Nobelpreises für Literatur
}

\author{
Eine linguistische Analyse
}

Zofia Bilut-Homplewicz (D) Maria Krauz $\mathbb{D}$

Eingegangen: 13. Februar 2021 / Angenommen: 16. September 2021 / Online publiziert: 2. März 2022 (C) Der/die Autor(en) 2022

Zusammenfassung Der Beitrag diskutiert das Konzept des liebevollen Erzählers, das Olga Tokarczuk in ihrer Nobelpreisvorlesung 2019 entwickelt hat. Es wird an die für die Nobelpreisträgerin wichtigen Werte erinnert und die Textaktualisierung dieser Kategorie analysiert. Darüber hinaus wird die Rolle des liebevollen Erzählers für die Interpretation der (textexternen) Wirklichkeit, für die Bewertung der für die zeitgenössische Welt wichtigen Erscheinungen sowie die mit dem liebevollen Erzähler verbundene Möglichkeit, Haltungen, Wahlentscheidungen und das Leben des zeitgenössischen Menschen zu beeinflussen, diskutiert. Da bei der Autorin eine mehrdimensionale Perspektive zum Einsatz kommt, machen wir auf Kontexte der Kategorien czuły und czułość nicht nur aus textorientierter, sondern auch aus einer breiteren, d. h. lexikologischen und semantischen Sicht aufmerksam. Die Analyse der Wörterbucheinträge von czuty und czutość sowie der Art und Weise, wie Tokarczuk den liebevollen Erzähler unmittelbar in der Rede oder in ihren weiteren Ausführungen aktualisiert und charakterisiert, führt zu dem Schluss, dass es sich bei dem postulierten neuen Erzähler in der »vierten Person« nicht um eine grammatische Konstruktion handelt, sondern um die Kategorie eines > wunderbaren< Erzählers, der eine universale Geschichte webt. Ihm werden von der Nobelpreisträgerin folgende Attribute zugeschrieben: Der liebevolle Erzähler kann die Perspektive jeder Figur einnehmen, er sieht weiter und mehr, er kann die Zeit ignorieren und er ist sich der Bedrohungen in der zeitgenössischen Welt bewusst. Der Beitrag unternimmt darüber hinaus den Versuch, eine Antwort darauf zu finden, wie man diese Gestalt und Kategorie mit der Rolle vereinbaren kann, die der Nobelpreisträgerin zufolge die Li-

Zofia Bilut-Homplewicz $(\bowtie) \cdot$ Maria Krauz

Katedra Lingwistyki Stosowanej, Instytut Neofilologii, Uniwersytet Rzeszowski, Rzeszów, Polen E-Mail: zbilut@ur.edu.pl

Maria Krauz

E-Mail: mkrauz@ur.edu.pl 
teratur in der zeitgenössischen Welt spielen soll: in einer Welt, die gespalten ist, die vom Menschen unvermindert zerstört wird und in der die Geistigkeit verschwindet.

Schlüsselwörter Der liebevolle Erzähler · Erzähler · Erzähltheorie ·

Textinterpretation · Lexikologie $\cdot$ Czuły $\cdot$ Czułość

\title{
The Concept of the Tender Narrator in Olga Tokarczuk's Nobel Lecture
} Aspects of Linguistic Analysis

\begin{abstract}
The paper introduces the concept of the tender narrator presented in her Nobel Lecture »The Tender Narrator« by Olga Tokarczuk. Reminiscent of the values important to the Nobel Prize winner, the paper analyzes the textual revision of this category. The subject of the discussion is also the role of the narrator in interpreting reality, assessing the phenomena in the contemporary world, as well as the possibility of the tender narrator to influence the attitude, choices and life of contemporary man. Given that the writer's own approach is multidimensional, the paper considers the contexts of the categories: tender and tenderness not only from textological, but also more broadly, from lexicological, semantic and cultural perspectives. The analysis of the lexemes czuty und czułośc as well as the ways of revising the tender narrator in the text of the lecture, expressed either directly or indirectly, based on the way the argument proceeds, leads to the conclusion that the postulated new, »fourth person « narrator is not just a grammatical construct. This is a wonderful storyteller who relates a universal, holistic and understandable story rooted in nature, who weaves an absolutely unique pattern. The Nobel Prize winner ascribes the tender narrator the following attributes: s/he (such narrator) knows how to embody the perspective of each person, how to see more broadly, how to ignore time, and is aware of the dangers of the contemporary world. Furthermore, the paper attempts to answer the question how to reconcile such person or category with the role that literature - according to the Nobel Prize winner - should play in the contemporary world; a world which is currently being destroyed by humans, divided into separate parts, and in which our spirituality is disappearing.
\end{abstract}

Keywords The Tender Narrator · Narrative Theory · Text Interpretation • Lexicology $\cdot$ Czuły $\cdot$ Czułość

\section{Vorbemerkungen}

Der Beitrag setzt sich mit dem Vorlesungstext von Olga Tokarczuk anlässlich der Verleihung des Nobelpreises für Literatur auseinander. Darin entwickelt die Preisträgerin - und der Titel kündigt es bereits an - für die Literatur das Schlüsselkonzept des liebevollen Erzählers und versucht es der Welt-Öffentlichkeit von Stockholm aus bekanntzumachen ${ }^{1}$.

\footnotetext{
1 Der Nobelpreis wurde der Schriftstellerin 2019 für das Jahr 2018 verliehen. Die Vorlesung anlässlich der Verleihung hat sie im Dezember 2019 gehalten.
} 
Der ursprüngliche Gedanke unserer Untersuchung war, dieses Konzept in erster Linie aus textlinguistischer Perspektive zu beleuchten. Es erwies sich aber als notwendig - was bei einem solchen Vorhaben durchaus als begründet erscheint, denn die Perspektive der Autorin ist bekanntlich breit angelegt -, weitere Gesichtspunkte, nämlich lexikologische, semantische sowie solche der Erzähltheorie ${ }^{2}$ mit zu berücksichtigen, wobei wir uns bewusst sind, dass die Interpretationsoffenheit, die ein Text besitzt, auch durch das Interesse des/der Rezipient*in determiniert ist. Auch Tokarczuk schreibt der Interpretation eine große Rolle zu, wenn sie in einem Essay über ihre Leseerfahrungen konstatiert: »Interpretation ist nicht eine andere Perzeption, sondern eine Sinnverleihung. « (Tokarczuk 2020d, S. 113)

Das Thema einer Vorlesung anlässlich der Verleihung des Nobelpreises weckt immer großes Interesse, nicht nur unter Schriftsteller*innen und Literaturwissenschaftler*innen, sondern überhaupt in der Welt der Kultur und Politik, weil - wie zu Recht angenommen wird - ein/eine Nobelpreisträger*in eine universale Botschaft zu verkünden hat. ${ }^{4}$ So auch im Falle von Olga Tokarczuk, die in ihrer Vorlesung erstmals ihr Konzept des >liebevollen Erzählers < für die Literatur öffentlich macht. ${ }^{5}$ Ihre Vorgehensweise, das Thema vorzustellen und zu entwickeln, ist dabei nicht weniger aufschlussreich als die Schlüsselinstanz selbst. Hellhörig macht allein schon die Kollokation czuty narrator (>der liebevolle Erzähler<), die weder im Polnischen noch im Deutschen bislang gebräuchlich war. ${ }^{6}$ Dabei sind die von Olga Tokarczuk geprägte Kategorie czułość (>Zärtlichkeit<) und das Konzept czuły narrator (>der zärtliche Erzähler $</>$ der liebevolle Erzähler $<^{7}$ ) nach Ryszard Nycz »revolutionäre Ideen, die das Potenzial besitzen, uns richtig durcheinander zu bringen und traditionelle Vektoren unserer Haltungen und Dispositionen zum Guten zu wenden: Denn: Ist Zärtlich-

\footnotetext{
2 Aspekte der Erzähltheorie werden hier nur am Rande Berücksichtigung finden, weil die von Tokarczuk vorgeschlagene Kategorie >der zärtliche Erzähler` nicht als eine feste Kategorie oder als oder als fixer Terminus im Sinne der literaturwissenschaftlich fundierten Erzähltheorie zu betrachten ist, sondern aus der Perspektive der Schriftstellerin, die nach neuen Wegen in der Literatur sucht, über die zeitgenössische Welt zu erzählen und den/die Leser*in zu sensibilisieren.

3 Übersetzt von Z. B.-H.

4 Vgl. den neuesten Beitrag von Wojciech Kajtoch (2021). Ihm zufolge wurde die Nobelpreisrede als Textsorte bisher nicht untersucht. Der Autor schreibt ihr drei Eigenschaften zu: Die in ihr behandelten Fragen sollen universal sein, wichtig für die Menschheit und wichtig für die jeweilige Zeit, zudem auch mit dem Gebiet verbunden sein, das der/die Nobelpreisträger*in repräsentiert, z. B. soll der/die Schriftsteller*in sich auf die Literatur beziehen.

5 Der Text mit dem Titel »Czuły narrator« (Tokarczuk 2019) ist zuerst unter https://xiegarnia.pl/ aktualnosci/olga-tokarczuk-mowa-noblowska/ (15.10.2020) erschienen, dann als Schlussbeitrag (Tokarczuk 2020b) des gleichnamigen Buches (2020a).

${ }^{6}$ Davon wird im Kapitel 2 die Rede sein.

7 In der im Kampa Verlag: Zürich 2020 veröffentlichten Vorlesung »Der liebevolle Erzähler«, die von Lisa Palmes aus dem Polnischen ins Deutsche übersetzt wurde, werden die Bezeichnungen liebevolle Zuneigung und der liebevolle Erzähler verwendet, die der vorliegende Beitrag beibehält. Auf die Bezeichnungen zärtlich, Zärtlichkeit und der zärtliche Erzähler wird immer dann zurückgegriffen, wenn die linguistischen Sachverhalte um die polnischen Wörter, Ausdrücke und Begriffe czuty, czułość und czuły narrator im Einzelnen dargelegt werden. Sie werden u. E. dem polnischen Original, sprich der ursprünglichen Vorlesungsfassung, eher gerecht. Aus Platzgründen muss eine Diskussion der Übersetzungsaspekte hier ausgeklammert werden. Die Übersetzung dieses Zitats - Z. B.-H.
} 
keit nicht all dem wohlgesinnt, was für die Existenz (im individuellen sowie im planetarischen Bereich) als gut gilt? $\ll^{8}$

\section{Einige Aspekte zum Leben und Schaffen von Olga Tokarczuk}

Im ersten Schritt des Beitrags wollen wir auf einige Aspekte des bisherigen Lebens und Schaffens der Schriftstellerin aufmerksam machen. Anschließend soll die textuelle Einbettung der Wörter, Ausdrücke und Begriffe wie czuty, narrator, czuty narrator und czułość sowie ihre Rolle bei der Interpretation der Wirklichkeit, bei der Bewertung der heutigen Welt verdeutlicht werden. Daneben soll auch die Möglichkeit des liebevollen Erzählers, Haltungen der Menschen zu beeinflussen, Gegenstand der Betrachtung sein.

Krzysztof Uniłowski macht darauf aufmerksam, dass nach einer Zeit anfänglicher Zurückhaltung und Distanzierung der literarischen Kritik gegenüber Tokarczuk die Zeit der spektakulären Gesten ihrer »Heiligsprechung « kam (vgl. Gołek-Sepetliewa 2020). Literaturkritiker*innen und Leser*innen heben hervor, dass Tokarczuks Schaffen durch eine reiche Vorstellungskraft gekennzeichnet sei ${ }^{9}$, dass ihr die Welt ihrer Protagonisten viel bedeute (vgl. beispielsweise Tokarczuks Vortrag »Psychologia narratora « 2020e) und dass die Schriftstellerin nach neuen Antworten auf Fragen suche, die mit schwierigen Problemen der Gegenwart verbunden sind, aber dass sie auch »aus vielen mythologischen Traditionen der Welt schöpft, angefangen mit dem Judentum und dem Christentum, über den Glauben der Slawen bis zu Jungs Psychoanalyse.«(Kozłowska 2020, S. 1) Dabei offenbare sie ihre persönlichen Gedanken und Erfahrungen, auch um »die Grenzen des Geistigen, die in Natur, Materie und Menschen eingebettet sind, zu entdecken« (Nalepa 2013, S. 133).

Es fehlt aber auch nicht an kritischen Stimmen. Sie betreffen vor allem den Anfang der schriftstellerischen Tätigkeit Tokarczuks. Betont werden u. a. kulturhistorische Ungenauigkeiten, eine populäre Art und Weise, Ausführungen zu gestalten, eine zu starke Transparenz der Sprache oder Schmeicheleien gegenüber dem Geschmack der Leserschaft (vgl. beispielsweise Kantner 2019).

Es wird darauf hingewiesen, dass Tokarczuk an die kreative Macht des Wortes glaubt, dass sie »das Wort sehr ernst nimmt, sich dem kabbalistischen Glauben und seiner Wirkkraft nähert und es als ein verstecktes Gewebe der Wirklichkeit betrachtet« (vgl. Kantner 2019, S. 10). Tokarczuk selbst, die sich mehrmals zur Literatur

\footnotetext{
8 Diese im Zitat des prominenten polonistischen Literaturtheoretikers enthaltene Wertung wurde auf dem Cover des Bandes »Czuły narrator« (2020a) platziert und ist als eine Art Werbung anzusehen. Der Band enthält 12 Essays und Vorträge Tokarczuks, die zwischen 2008 und 2020 entstanden sind. Es ist das erste Buch, das die Autorin nach der Verleihung des Nobelpreises veröffentlicht hat. Manche dieser Texte wurden bereits früher publiziert. Tokarczuk (ebenda, S. 292) schreibt am Ende des Bandes in einer Anmerkung, dass sie ihn im gewissen Sinne der Pandemie verdankt, weil sie gerade in dieser Zeit die Notwenigkeit gesehen hat, ihre Texte zu ordnen.

9 Das Buch Der liebevolle Erzähler, dem, wie bereits angemerkt, der hier analysierte Text entnommen ist, eröffnen folgende Worte der Schwedischen Akademie: »Der Nobelpreis für Literatur 2018 wird verliehen an Olga Tokarczuk für eine erzählerische Vorstellungskraft, die mit enzyklopädischer Leidenschaft das Überschreiten von Grenzen als Lebensform symbolisiert«.
} 
und zur Poetik des Romans geäußert hat, schlägt einen Kommunikationspakt mit den Leser*innen vor: »Wir gehen nicht davon aus, dass die Kunst unverständlich und unzugänglich ist, dass sie durch großen Ehrgeiz und kühle Distanz geprägt ist, wir gehen davon aus, einen solchen Kontext der Geschichte zu schaffen, in dem wir uns Rücken an Rücken lehnen und uns das Schicksal vorhersagen werden « (nach Kącka 2020, S. 13). Dieser Ausgangspunkt ist wichtig, weil das Schaffen der Nobelpreisträgerin, wie Eliza Kącka schreibt, eine Rückkehr zu den Wurzeln bedeutet; es ist die Stimme der Schriftstellerin, der Mitgestalterin der kollektiven Vorstellungskraft, die Stimme der Erzählerin im Hintergrund der Stimmen anderer Heilerinnen (Kącka 2020, S. 11).

Es stellt sich mithin für diejenigen, die eine Analyse der Texte dieser Schriftstellerin wagen, eine sehr anspruchsvolle Aufgabe. Eine Analyse der Vorlesung in toto und des von der Schriftstellerin holistisch angelegten Plädoyers, die Relation zwischen Literatur und Welt zu gestalten ${ }^{10}$ und über sie so zu erzählen, dass damit Einfluss auf die sich in tiefer Krise befindende Welt ausgeübt werden kann, vermag ein/eine Linguist*in nur partiell zu leisten. Im Falle der Nobelpreisrede handelt es sich zwar nicht um einen literarischen Text im engeren Sinn, sondern um eine essayistisch orientierte Vorlesung, aber seine Prägung durch die Schriftstellerin-Autorin ist doch sehr deutlich, was ihn zweifellos zu einem sprachlich und inhaltlich anspruchsvollen Stück Wortarbeit macht. ${ }^{11}$

\section{Die Lexeme czułość und czuły aus lexikologischer Sicht}

Es scheint angeraten, einer linguistischen Analyse des Konzepts des liebevollen Erzählers einige lexikologische Bemerkungen zu den im Titel des Kapitels erwähnten Lexemen voranzustellen, weil sie für unser Vorhaben von grundsätzlicher Bedeutung sind. Wir konzentrieren uns in erster Linie auf die polnischen Lexeme, weil der Ausgangstext auf Polnisch verfasst wurde, für den diese lexikalischen Einheiten den Interpretationsrahmen eröffnen. ${ }^{12}$

Als Derivationsbasis für die Derivate czucie, czułość, czuły, czulić się, czułki, czujny gilt das slawische Verb czuć (urslawisch: čuti) (>fühlen $<$ ), das bereits im 14. Jahrhundert vorkommt. Im etymologischen Wörterbuch von Długosz-Kurczabowa (2006) wird es folgendermaßen definiert: »Sinneseindrücke erfahren, Gefühle erfahren, etwas innerlich erleben, sich etwas bewusst werden«; das Adjektiv czuły (aus

\footnotetext{
10 Darauf wird in Kap. 5 eingegangen.

11 Am Rande angemerkt sei, dass die Analyse literarischer Texte zur gängigen Praxis in der polonistischen Textlinguistik gehört, was in germanistischen Untersuchungen nicht der Fall ist. Dies ist auf die unterschiedliche Forschungstradition der beiden Schreibkulturen zurückzuführen; vgl. beispielsweise Dobrzyńska (1974, 1993), Witosz (2005) - Übersetzung ins Deutsche (2015), Filip/Krauz (2010), BilutHomplewicz (2013). In ihrem vielzitierten Beitrag zur Zukunft der Textsortenlinguistik plädiert Kirsten Adamzik (2001) jedoch für die Untersuchung von anspruchsvollen und komplexen Texten im Rahmen einer kontrastiven Textologie.

12 Dadurch kann der/die deutschsprachige Leser*in auf einige Nuancen des Originals aufmerksam gemacht und für sie sensibilisiert werden.
} 
dem Ursl. čul) hat die Bedeutungen czujny, wyczulony, wrażliwy (ebenda, S. 92) ( >aufmerksam<, > wachsam<, >empfindlich<, > sensibel<). ${ }^{13}$

Für jede Bedeutungsvariante von czuty gibt es einige Synonyme:

1. czuty (bezogen auf die Person), z. B. Geliebter, Sohn - >zärtlich <, >liebevoll<, > herzlich<;

2. czuty (Stimme, Blick) - >sanft<;

3. czuty (auf Ungerechtigkeiten, auf Schönheit) - >empfindlich<;

4. czuty (Mechanismus) - >fein<, > genau< (vgl. Bańko 2008, S. 110). ${ }^{14}$

Die Vielfalt der Bedeutungsvarianten hat zur Folge, dass die Lexeme czuły/czułość eine allgemeine und weit gefasste Bedeutung verbindet: Sensibilität, Liebe, Wärme und Sorge ausstrahlend. Die Analyse der Gebrauchskontexte ${ }^{15}$ des Lexems czuty zeigt, dass es in bestimmten Ausdrücken vorkommt: czuty $i$ wrażliwy (>zärtlich und sensibel<), czuty i opiekuńczy (>zärtlich und fürsorglich<), czuty i serdeczny (>zärtlich und herzlich<), czuty i troskliwy (>zärtlich und sorgsam<), czuty i delikatny (>zärtlich und empfindsam<). Czułość also kommt die Bedeutung einer wichtigen Kategorie zu, deren Bestimmung die philologische Tiefensonde erfordert, um möglichst alle Bedeutungsschattierungen ans Licht zu befördern. Alle diese Eigenschaften beziehen sich auch auf den liebevollen Erzähler, der, wie schon erwähnt, ein immenses Wissen besitzt, alles versteht, viel erlebt, die Welt interpretiert, Zeitgrenzen transzendiert, der mehr als die anderen, ja sogar alles sieht und die Erlebnisse der Protagonisten mitempfindet und nachvollzieht. ${ }^{16}$ An dieser Stelle drängen sich einige Fragen auf: Hat er auch die Kraft, den Menschen und die Welt zu beeinflussen? Wie (re)konstruiert Olga Tokarczuk die Kategorie des liebevollen Erzählers? Welche Vorstellungswelt wird in der Vorlesung evoziert? Es werden im Folgenden Antworten dazu gesucht, die sich auf die (text)linguistische Analyse stützen.

Was die Wiedergabe dieser lexikalischen Einheiten in der deutschen Übersetzung anbelangt, so kommt im polnischen Text die Verbindung zwischen czutość und czuty in der Wortbildung deutlich zum Ausdruck. Das Adjektiv czuty stellt eine Wurzel zur Bildung des Substantivs czułość dar, was man mit dem Adjektiv zärtlich und seinem Derivat Zärtlichkeit verdeutlichen könnte. Das deutsche Lexem zärtlich würde deshalb mit dem Substantiv Zärtlichkeit als Derivat eine analoge Relation zwischen den beiden Wörtern wiedergeben. Dies wird in der deutschen Übersetzung mit dem Lexem >liebevoll< und der Wortgruppe >liebevolle Zuneigung < nicht erreicht, so dass

\footnotetext{
13 Die polnischen Entsprechungen wurden in Wiktorowicz/Frączek (2008) und unter https://en.pons.com/ translate/german-polish/z\%C3\%A4rtlich (10.12.2020) nachgeschlagen.

14 Vgl. die Anm. 8.

1. czuty (w odniesieniu do osoby), np. kochanek, syn - kochajacy, tkliwy, pieszczotliwy, serdeczny;:

2. czuły (głos, spojrzenie) - miękki, delikatny;

3. czuły (na krzywdę, piękno) - wrażliwy;.

4. czuty (mechanizm) - dokładny (Bańko 2008, S. 110).

15 Vgl. »Narodowy Korpus Języka Polskiego Pelctra« (12.11.2020).

16 Diese Charakteristik ergibt sich aus dem Inhalt des Gesamttextes.
} 
für die deutschsprachigen Leser*innen der Charakter der polnischen Kollokation czuty narrator in gewissem Sinne verlorengeht. ${ }^{17}$

In diesem Zusammenhang auch noch eine Anmerkung zu Kollokationen: Wie die Analyse der Kontexte zeigt ${ }^{18}$, kommt das Lexem narrator (〉Erzähler $<$ ) in Verbindung mit dem Adjektiv czuły nicht vor, diese Kollokation ist nicht gebräuchlich. Üblich sind andere polnische Lexeme, die als Kollokationen auftreten, beispielsweise bezogen auf opowiadanie, ksiażka, historia, pamiętniki (>Erzählung<, >Buch<, $>$ Geschichte<, >Tagebücher<) (mit dem Genitiv). Das Adjektiv czuły kommt dagegen oft mit Substantiven vor, die als Personenbezeichnungen fungieren, beispielsweise kochanek, meżczyzna, kobieta, matka (>Geliebter<, >Mann<, >Frau<, >Mutter $<$ ) oder mit Bezeichnungen der Sinne: dotyk, głos, wzrok ( $>$ Tastsinn $<, ~>S t i m m e<, ~>S e h k r a f t<)$. Es kann sich somit laut des angeführten Wörterbuchseintrags und der darin angegebenen Kontexte um Kollokationen mit solchen Wörtern wie stowa (>Worte $<$ ), pozdrowienia (>Grüße $<$ ), powitania ( $>$ Begrüßungen $<$ ) oder listy (>Briefe $<$ ) handeln. Die Kollokation des Lexems czuty und des literaturwissenschaftlichen Terminus Erzähler ist somit originell. Man kann ihn auf den czuty obserwator (>der aufmerksame Beobachter $</>$ Betrachter $<$ ) beziehen, besonders dann, wenn der Erzähler als ein wichtiges Element der Erzählsituation angesehen werden kann, als Subjekt der Narration.

\footnotetext{
${ }^{17}$ Es sei hier nur am Rande darauf hingewiesen, dass in dem Artikel »Nobelpreisrede. Tokarczuks Plädoyer für die Sensibilität«, in: https://orf.at/stories/3146807/ (12.12.2020), interessanterweise die Bezeichnungen Sensibilität und der sensible Erzähler verwendet werden. In einem anderen Presseartikel erscheint dagegen im Titel die Wortgruppe »Auf der Suche nach der zärtlichen Erzählinstanz« mit dem Adjektiv zärtlich - während man im Text sowohl die Synonyme verwendet (»Ihre gut einstündige, auf Polnisch gehaltene Vorlesung hat Olga Tokarczuk »Czuły narrator« [»The Tender Narrator«] genannt, also der »zärtliche« oder »liebevolle« Erzähler.«) als auch nur das Adjektiv zärtlich (»dass eine junge Frau, die niemals religiös war - meine Mutter - mir so etwas wie eine Seele gab und mich damit zugleich mit dem weltgrößten zärtlichen Erzähler versah«), schloss Tokarczuk diese sehr persönliche poetologische Eingangspassage.« (Hillgruber [2019], vgl. https://www.tagesspiegel.de/kultur/die-nobelpreis-rede-von-olga-tokarczuk-aufder-suche-nach-der-zaertlichen-erzaehlinstanz/25312238.html (12.12.2019).
}

$18 \mathrm{Vgl}$. »Wielki słownik języka polskiego«: https://www.wsjp.pl/ (12.12.2020). 


\section{Kategorien der Erzählung und des Erzählers}

In welcher Beziehung steht nun der liebvolle Erzähler zu den Erzähltypen der Erzähltheorie? ${ }^{19}$ Es muss gleich vorausgeschickt und hervorgehoben werden, dass es nicht das Ziel von Tokarczuk ist, einen literaturwissenschaftlichen Beitrag zur Erzähltheorie zu leisten, etwa eine neue Typologie des Erzählers anzustreben, auch wenn sie das grundlegende Begriffsinstrumentarium der Erzähltheorie, d. h. die Bezeichnungen >Ich-Erzähler $<$, > Erzählen $<$, >Fabel $<$ und $>$ Fiktion $<$ in der analysierten Vorlesung im allgemeinen Sinne benutzt und zu bedienen versteht.

Die Kategorie des Erzählers wurde von Tokarczuk bereits in einem früheren Vortrag mit dem Titel »Psychologia narratora $\ll^{20}$ ( $>$ Psychologie des Erzählers $<$ ) im Jahr $2018^{21}$ behandelt - und damit offensichtlich der Weg zu dem hier im Mittelpunkt der Analyse stehenden liebevollen Erzähler aus der Nobelpreisrede angebahnt. Es ist derselbe Erzähler, der in der Nobelpreisvorlesung als liebevoller Erzähler oder Erzähler in der »vierten Person « (narrator czwartoosobowy) firmiert. ${ }^{22}$ Es handelt sich bei der Anknüpfung aber nicht um grammatische Eigenschaften, die überein-

19 Eine Synthese verschiedener Ansätze und Aspekte der Erzähltheorie ist bei Piotr Kulas (2014) zu finden. Der Autor weist zu Recht auf die Unterschiedlichkeit und Interdisziplinarität der Narrationsforschung in vielen geisteswissenschaftlichen Disziplinen hin, unter anderem in der Literaturwissenschaft, in den Sozialwissenschaften, der Psychologie, der Philosophie und in den Kulturwissenschaften. Die Narration wird beispielsweise als Erzählung eines einzelnen Subjekts oder einer Gruppe, als fertiger Text ebenso wie als Erkenntnisstruktur oder Struktur des Verstehens verstanden.

Es wird hier darauf verzichtet, die Ergebnisse der Erzählforschung vorzutragen und zu diskutieren sowie Modelle des Erzählers und der Erzähltheorie darzustellen. Interessierte finden Auskunft in inzwischen klassischen Werken, beispielsweise in dem von Franz K. Stanzel (2008, 8. Aufl.): Dabei geht es um die auktoriale Erzählsituation mit einem allwissenden Erzähler, der sich nicht neutral zur Handlung verhält und sich immer wieder kommentierend und bewertend in die Handlung einmischt, oder um die personale Erzählsituation, bei der es sich um die Erzählung aus der Sicht einer bestimmten Figur handelt, schließlich um die Ich-Erzählsituation, bei der in der ersten Person erzählt wird. Auch der Ansatz von Gérard Genette (2010): »Die Erzählung«, der das Verhältnis zwischen dem Wissen eines Erzählers und dem einer Figur beschreibt und die Termini >Nullfokalisierung<, >interneく sowie >externe Fokalisierung prägt, gehört zum Kanon der Erzählforschung.

$\mathrm{Zu}$ erwähnen ist darüber hinaus die Studie von Matias Martinez und Michael Scheffel: »Einführung in die Erzähltheorie« (2016), die bereits zehn Auflagen erlebt hat. Die beiden Autoren behandeln außerdem solche Kategorien wie >Zeit<, >Modus $<$ und >Stimme<, setzen sich mit Franz K. Stanzels Typologie von Erzählsituationen kritisch auseinander und gehen auf Größen wie Handlung, erzählte Welt, Figur und Raum ein. Einige polonistische Arbeiten sind ebenfalls zu nennen. Vgl. Henryk Markiewicz (1984), der zum Vorrang der Narration in einem epischen Werk über die Ereignisse schreibt (der ontologische Vorrang, der temporale Vorrang). Mit Teun van Dijk (1985, S. 145 ff.) ist festzuhalten, dass Narration seit Jahrhunderten mit literarischen Texten verbunden war, heute jedoch nicht nur auf künstlerische Narrationen zu beziehen ist, sondern auch auf diejenigen, die das natürliche Gespräch betreffen. Auch Jacek Warchala (1993) sucht die Exponenten der Narration im Dialog.

Der Terminus >Journalistische Narration « wird dagegen von Aleksander Wilkoń (2002, S. 124) verwendet. Beata Grochala (2013, S. 237) spricht von Narration im Hinblick auf das Fußballspiel und unterscheidet zwischen der Makro- und Mikronarration. Auch für Franz K. Stanzel (1993) ist der Erzähler nicht nur mit literarischen Erzählungen verbunden. Ihm zufolge sind wir alle als Erzähler*innen zu betrachten, auch wenn wir keine epischen Werke verfassen. Wir sind es in Gesprächen, Berichten und vor allem in unseren Erinnerungen. (vgl. Grochala 2013, S. 237).

$20>$ Psychologie des Erzählers $<$. Übrigens ist Tokarczuk ausgebildete Psychologin.

21 Tokarczuk hat den Vortrag 2018 an der Universität Łódź gehalten, er wurde in Tokarczuk (2020a) unter selbigem Titel veröffentlicht.

22 In »Psychologia narratora« erscheint nur die Bezeichnung Erzähler in der vierten Person. 
stimmen würden, sondern um die Art und Weise, die Welt zu empfinden sowie um die Schaffenskraft, d. h. um die potentielle Wirksamkeit, die Handlung des Werkes zu gestalten.

Auf eine spannende Art und Weise macht die Autorin in diesem Text ihre Leserschaft mit den verschiedenen Typen des Erzählers bekannt. Sie widmet sich dieser Frage aus ihrer Erfahrung als Schriftstellerin heraus, nicht aus der Sicht der Literaturwissenschaft, und führt zahlreiche Beispiele, vor allem aus dem eigenen Schaffen, aber auch aus anderen epischen Werken an, wobei sie auf den Zusammenhang zwischen der Art der Geschichte und dem jeweiligen Erzähler hinweist. Tokarczuk selbst charakterisiert den Erzähler in »Psychologia narratora « folgendermaßen: »Es ist etwas, was im Schlaf versunken ist, das seine Kraft offenbart wie ein nichtaktives Gen (...)«(2020e, S. 155). Aus diesem Zitat ist deutlich zu ersehen, dass hier die Perspektive der Literatur, die auf der Vorstellungskraft beruht und nicht literaturwissenschaftliches Wissen aktivieren soll, im Spiel ist. Gleich am Textanfang wird die Rolle des Erzählers angesprochen, wenn Tokarczuk konstatiert, dass für das Schreiben einer guten Geschichte das beste Thema, sorgfältige Recherchen und gute Arbeitsbedingungen nicht ausreichend sind, man brauche stets den Erzähler. Für die Autorin stellen ihre Überlegungen zum Erzähler und zu seiner Gestaltung einen langwierigen Prozess dar, der beim Schreiben des Romans »Dom dzienny, dom nocny« (»Taghaus, Nachthaus«) begonnen hat. Sie bezeichnet ihre Bemühungen und Erfahrungen um die Gestaltung der Erzählfunktion als ćwiczenia z narratora ${ }^{23}$ (>Übungen zum Erzähler<), als ihr Abenteuer mit potenziellen Erzählern. Sie hat dabei die neue Bezeichnung narrator panoptykalny - niezwykty, dziwny (>außerordentlich $<$, > ungewöhnlichく, >panoptisch $<$ ) geprägt - eine unpersönliche Erzählinstanz im Text, deren Perspektive und Wissen praktisch unbegrenzt sind (ebenda, S. 170).

Es ist somit die Rede vom allwissenden Erzähler, den Tokarczuk als Leserin liebt. Sie merkt dabei jedoch an, dass er von dem/der Autor*in eine Yoga-Konzentration verlangt. Als Gegenpol nennt sie den Ich-Erzähler, der sich emotional an der Handlung beteiligt.

»Psychologia narratora « berechtigt zu der folgenden Schlussfolgerung: Wenn man versuchen würde, das Konzept des liebevollen Erzählers erzähltheoretisch zu fassen, müsste man sagen, dass es nicht zu leisten ist, weil sich die Schriftstellerin nicht auf der Ebene der Erzähltheorie bewegt, sondern nur bestimmte ihrer Elemente benutzt. Tokarczuk strebt, wie gesagt, keine Erzähltypologie an. Die Gemeinsamkeit mit der Erzähltheorie von Genette könnte man lediglich darin sehen, dass der Erzähler in der vierten Person bei Tokarczuk als Figur vorkommen kann (wie Jenta im monumentalen Roman »Die Jakobsbücher«) oder nicht. Interessanterweise sind in diesem Werk bereits andere Erzähler - der dissoziative Erzähler, der sich von der Autorin löst, der Er-Erzähler, der sich von ihr distanziert, also der autonome Erzähler - vorhanden, die der Schriftstellerin jedoch nicht ausreichen. »In einem Moment des Schreibens fühlte ich, dass ich ratlos dastehe und dass ich bereits alle Möglichkeiten, die Narration bietet, genutzt habe.« (ebenda, S. 176) berichtet Tokarczuk. Es zeigte sich, dass in einem so komplexen Werk noch eine Erzählstimme notwendig war, die die Zeit-

${ }^{23}$ Die polnische Phrase wird so formuliert, als ob es sich um ein Schulfach handeln würde, in dem Übungen gemacht werden, wie beispielsweise ćwiczenia z matematyki (>Übungen in Mathematik`). 
grenzen überschreitet und alles von oben betrachtet. Jenta, eine der Hauptfiguren, wird zu einer solchen Erzählerin. Sie übernimmt diese Stimme. Die Schriftstellerin spricht in ihrem Fall von der Erzählerin in der vierten Person (narratorka czwartoosobowa), was mit der Bezeichnung und Rolle des liebevollen Erzählers in der analysierten Nobelpreisvorlesung koinzidiert, wo er jedoch als zentrales Konzept viel ausführlicher charakterisiert wird.

Die von Tokarczuk genannten Typen von Erzählern sind in erster Linie auf ihre Erfahrungen, die sie beim Verfassen ihrer konkreten Werke gemacht hat, zurückzuführen. Die Schriftstellerin sagt, dass sie beim Verfassen des hier behandelten diskutierten Romans »Księgi Jakubowe « immer wieder ratlos war, weil sie bereits alle erzählerischen Möglichkeiten im Hinblick auf die komplizierte Handlung ausgenutzt zu haben schien und vor dem Problem stand, das Werk als Ganzes zu schaffen. Die Schriftstellerin fasst es bildhaft wie folgt: »Als ich in tiefe Verzweiflung bei der Betrachtung des unfertigen Textes geriet, tauchte Jenta ruck zuck wie ein Flaschengeist aus Aladins Wunderlampe auf - eine Figur, deren physische und psychische Grenzen nicht mehr nach menschlichem Maß sind. Dank dessen schaut Jena durch die Zeit und sieht alles von oben.«(ebenda, S. 176f.)

Man könnte hier bestimmte Züge des auktorialen Erzählers im Sinne von Stanzel (2008) oder der Nullfokalisierung im Sinne von Genette (2010) ausmachen, aber es würde sich dann um keine klare Zuordnung handeln, weil es nicht die Absicht Tokarczuks ist, eine Typologie zu erstellen. Die Vagheit der Erzählerqualität resultiert vielmehr aus der Tatsache, dass hier zwei Ebenen im Spiel sind - nämlich die der literaturwissenschaftlichen Terminologie und der damit zusammenhängenden Sachverhalte einerseits und die der Welt der Schriftstellerin anderseits -, in der die gängigen Begriffe des >Ich-Erzählers < und der >Erzählung< zwar erscheinen, in der jedoch der liebevolle Erzähler als Tokarczuks schriftstellerische Erfindung gilt.

\section{Die sprachliche Manifestation des liebevollen Erzählers und der liebevollen Zuneigung im Vorlesungstext}

\subsection{Das Konzept czuły narrator (>der liebevolle Erzähler $<$ )}

Im Folgenden geht es um die explizite Erscheinungsweise des liebevollen Erzählers im Sinne seiner Nennung und seiner impliziten Thematisierung verdeutlicht werden.

Bereits die erste Lektüre des Textes macht deutlich, dass dem >liebevollen Erzähler (als Kategorie!) im bzw. als Titel eine strategische Position zukommt; auch wird er gleich im Schlusssatz des ersten Kapitels explizit erwähnt. Ohne Zweifel eröffnet die Verwendung und somit die Thematisierung dieser Kollokation eine im Hinblick auf den weiteren Textablauf kataphorische Erwartung, die mit der Frage zusammenhängt, wie diese Instanz von Tokarczuk aufgefasst und welche Rolle ihr zugeschrieben wird. Im Textganzen und am Schluss des Textes kommt dagegen das Verb erzählen als Manifestation einer umfangreichen Isotopiekette vor. Im Titel erscheint die bloße Bezeichnung der liebevolle Erzähler ohne weitere begleitende sprachliche Elemente, was die Prägnanz dieses Ausdrucks verstärkt und ihm eine 
aufmerksamkeitssteuernde Funktion verleiht, die damit zugleich in den Mittelpunkt rückt und als zentrales Konzept des Gesamttextes gilt.

Gleich in der Eingangspassage wird ihm eine besondere Rolle zugeschrieben. Als Schöpferin fungiert die Mutter der Autorin, die durch ihre emotionale Kraft in der Lage ist, dem nicht Existierenden in gewissem Sinne zur Existenz zu verhelfen. Sie erklärt ihrer kleinen Tochter, dass, als sie noch nicht auf der Welt war, sie bereits von ihr vermisst wurde. Tokarczuk drückt es so aus, dass ihre Mutter, eine junge, nichtreligiöse Person, ihr »den liebevollsten Erzähler der Welt zur Seite [stellte].« (S. 14)

Damit kommt eine Überzeugung zur Sprache, dass das Gefühl des Vermissens nicht nur mit einem konkreten Verlust verbunden sein muss, sondern dass man auch jemanden vermissen kann, den es noch nicht gibt. Die Mutter erklärt: »Wenn man jemanden vermisst, bedeutet das, dieser Jemand ist schon da.« (S. 13) Die Nobelpreisträgerin greift nicht zufällig auf die Beziehung zur Mutter gleich am Anfang der Vorlesung zurück. Es geht dabei um den Prozess der Gestaltung der Persönlichkeit der Tochter in ihrer Kindheit, der die Mutter diese Geschichte erzählt und dadurch ihre Sensibilität anregt, was auch mit der Vorstellungskraft der Schriftstellerin in Verbindung steht. Es drängt sich bei dieser intensiven persönlich geprägten Äußerung eine Analogie auf, dass der noch nicht bekannte liebevolle Erzähler, der mit der Seele, d. h. mit einer geistigen Größe gleichgesetzt wird, auch eine besondere Kraft besitzen muss.

Der Ausdruck der liebevolle Erzähler wird dann im 6. Kapitel wieder aufgenommen. Als wichtig für die Interpretation der Vorlesung kann Tokarczuks Frage betrachtet werden, die direkt an die Bibel anknüpft und der sich drei weitere Fragen anschließen. Sie gehen dem Schlüsselbegriff der liebevolle Erzähler voran.

So zitiert Tokarczuk zitiert aus dem Johannes Evangelium: »Im Anfang war das Wort.« Diese rhetorisch geprägte Anknüpfung an die Bibel initiiert die eigentliche Einkreisung des liebevollen Erzählers.

»Haben Sie schon einmal gefragt, wer dieser wunderbare Erzähler (pl. cudowny opowiadacz, vgl. S. 52) ist, der in der Bibel mit weithin tönender Stimme verkündet: >Im Anfang war das Wort <? Der die Schöpfung der Welt beschreibt, ihren ersten Tag, an dem die Wirrnis von der Ordnung geschieden wird? Wer durchlebt sie, die Serie von der Entstehung des Kosmos? Wer kennt Gottes Gedanken, Gottes Zweifel, wer bringt mit sicherer Hand jenen außerordentlichen Satz zu Papier: >Gott sah, dass es gut war $<$ ? Wer ist es, der weiß, was Gott dachte?«(S. 52 f. $)^{24}$

Hier ist ebenfalls eine gewisse Analogie zum ersten Kapitel zu bemerken, in dem der liebevolle Erzähler mit einer äußerst positiven Wertung versehen und sogar superlativisch als »der liebevollste Erzähler« bezeichnet wird.

\footnotetext{
24 Auch wenn explizit auf die Bibel Bezug genommen wird, stellt Tokarczuk fest, dass sie sich nicht in theologische Diskussionen einlassen will: »Wenn wir alle theologischen Fragwürdigkeiten einmal beiseitelassen, erkennen wir, welch große Bedeutung dieser Figur zukommt, diesem mysteriösen und liebevollen Erzähler.« (S. 53).
} 
Bemerkenswert ist, dass die beiden Stellen in einen Kontext eingebettet sind, in dem Sachverhalte auftauchen, die das Immaterielle, das Geistige besonders kennzeichnen und herausstellen - der Erzähler erscheint zuerst als eine literarische Paraphrase der Seele (Kap. 1) und wird als Instanz entworfen, die offenbar die Gedanken Gottes kennen kann (vgl. oben, Kapitel 6). Eines steht fest: Die Transzendenz ist in beiden Passagen präsent.

Der liebevolle Erzähler erscheint jedoch nicht ausschließlich als eine neue Instanz. Er erwächst in erster Linie aus der Kontrastierung mit dem Ich-Erzähler, der im vorausgehenden 2. Kapitel thematisiert wird. Letzterem wird viel Aufmerksamkeit gewidmet, seine scheinbaren Vorzüge, vor allem aber seine Unzulänglichkeiten und Mängel werden deutlich gemacht. Hier wird die Suche nach einer neuen Erzählinstanz deutlich: Eben die Auseinandersetzung mit der Ich-Erzählung macht einen wesentlichen Teil des 2. Kapitels aus und stellt ein relevantes Teilthema des Gesamttextes dar. Die Wiederaufnahme dieses Teilthemas im 6. Kapitel der Vorlesung, übrigens das vorletzte Kapitel insgesamt, ist im Hinblick auf die Kohärenzstiftung als strategischer Zug zu werten. Tokarczuk stiftet Kohärenz mit dem Textganzen, indem sie die Rolle des Wortes und die des Erzählens als fundamental hervorhebt.

Das vorletzte Kapitel schließt mit einer Parallele an ein für die europäische Kultur fundamentales Werk an, und zwar »Doktor Faustus« von Thomas Mann, und einer seiner Figuren, dem Tonsetzer Adrian Leverkühn, der eine neue Tonart erfindet; diese besitze die Fähigkeit, die Welt zu verändern. »Was Mann jedoch nicht schildert, ist ihre Form; er schafft lediglich eine Vorstellung davon, wie diese Musik klingen könnte. ${ }^{25}$ Vielleicht besteht die Rolle des Künstlers eben genau darin - eine einen Vorgeschmack darauf zu geben, was sein könnte, und so dafür zu sorgen, dass es eben vorstellbar wird. Vorstellbarkeit ist schließlich die erste Stufe des Seins.« (57f.)

Auch hier drängen sich wieder Parallelen auf, und zwar zur Mutter, die ihre noch nicht geborene Tochter vermisst, und zum liebevollen Erzähler, was von besonderer Wichtigkeit ist. Noch nicht Existierendes kann dank der Vorstellungskraft seinen Anfang nehmen.

Des Weiteren ist beachtenswert, wie der liebevolle Erzähler implizit im Text dargestellt und charakterisiert wird. Diese Strategie ist von einem/einer aufmerksamen Leser*in zu entschlüsseln. Dem liebevollen Erzähler ist nämlich eine bestimmte Erzählweise eigen: Statt die Geschichte zu schreiben und zu erzählen, soll er an der bzw. die Geschichte >weben<; dieser Ausdruck kommt mehrmals in der Vorlesung zur Sprache. Er soll die Welt eines >Ich< betrachten, sein eigenes Schicksal erzählen, die Kreation >einer Stimme, die spricht< darbieten, eine Erzählung konstruieren, in der alles Wesentliche enthalten ist.

Emotionen betonen das reiche Innenleben des Erzählers, der an der Geschichte webt. Er ist eine Gestalt, die nicht nur alles weiß, sondern auch alles miterlebt, die durch eine reiche Vorstellungskraft gekennzeichnet ist, die es versteht, Empathie zu zeigen und die Vorstellungskraft der Leser anzuregen und zu entwickeln. Zum Ausdruck emotionaler Zustände dienen bekanntlich verbale Mittel, die im Medium der

25 Wieder muss auf eine bestimmte Unzulänglichkeit der Übersetzung hingewiesen werden. Im Original ist davon die Rede, dass nicht beschrieben wurde, worauf diese Musik beruhen könnte (Tokarczuk 2020b, S. 287), in der Übersetzung (Tokarczuk 2020c, S 57) dagegen ist von der Form dieser Musik die Rede. 
Schrift dominieren sowie nonverbale und paraverbale Mittel, die für direkte Kontakte charakteristisch sind. (vgl. Awdiejew 2007, S. 122; Awdiejew/Habrajska 2006, S. 10f.) In dem analysierten Text sind sowohl Bezeichnungen für Gefühle und Emotionen präsent, die in erster Linie das semantische Feld des Erlebens repräsentieren, als auch Ausdrücke, die die nichtverbale Intensivierung von Empfindungen wiedergeben, z. B. sich sehnen, fühlen, überlief mich (...) ein Schauer, mit heißen Wangen und Tränen in den Augen. All die Bezeichnungen beziehen sich auf die Schriftstellerin selbst, die ihre emotionalen Reaktionen charakterisiert und somit > vorführt<, wie der zärtliche Erzähler, den sie selbst verkörpern will, sprachlich handelt.

Derart konturiert sich ein sensibles Wesen, das durch profunde Erlebnisse gekennzeichnet ist und entsprechend intensiv auf die Wirklichkeit reagiert, eine scharfsinnige Beobachter- und sensible Schöpferfigur, wobei weitere Attribute des geschichtenwebenden Erzählers zu Tage treten. Die Nobelpreisträgerin gibt in ihrem Text Entwicklungsetappen ihres liebevollen Erzählers preis: das allseitige Beobachten, die Anregung der Vorstellungskraft, das Erleben, der Glaube, die Nähe und die Erinnerung an die Mutter.

Die sinnlich geprägte Auffassung der Welt wird auch in der folgenden initialen Passage spürbar, in der >die Geburt< des liebevollen Erzählers beschrieben wird:

»Das erste Bild, das ich bewusst wahrgenommen habe, ist ein Foto meiner Mutter noch aus der Zeit vor meiner Geburt. Leider ist es eine Schwarz-Weiß-Aufnahme, dadurch lassen sich viele Details schwer erkennen, lediglich als graue Schemen. Das Licht ist weich wie bei Regen, ein Frühjahrslicht ist es wohl, das durchs Fenster sickert und den Raum nur schwach erhellt. Meine Mutter sitzt bei dem alten Radio - es ist einer dieser Apparate mit grünem Auge und zwei Knöpfen, einem für die Regulierung der Lautstärke, einem für die Sendersuche. [...] Ich glaubte fest daran, dass durch das Radio andere Sonnensysteme und Galaxien zu mir sprachen und mir zwischen Knacken und Rauschen Botschaften sandten, die ich einfach nicht entschlüsseln konnte. [...] Die leicht gebeugt dasitzende Frau hat ihren Blick auf einen Punkt jenseits des Bildrandes gerichtet. Sie sieht etwas, was dem Betrachter verborgen bleibt. Als Kind meinte ich, sie betrachte die Zeit. Auf dem Foto ereignet sich nichts; es bildet einen Zustand ab, keinen Prozess. Die Frau wirkt traurig, in Gedanken versunken, abwesend. [...] Und so gab mir diese junge Frau, die nie religiös gewesen war - meine Mutter -, etwas, das man früher »Seele« nannte - und stellte mir damit den liebevollsten Erzähler der Welt zur Seite.« (S. 11-14)

Bei der ersten Lektüre scheint es die gewöhnliche, einfache Beschreibung eines alten Schwarz-Weiß-Fotos zu sein, aber es lässt sich ohne weiteres behaupten, dass hier der liebevollen Zuneigung Ausdruck verliehen wird. Ein Betrachter spricht nicht vom Sehen oder von der durch das Betrachten ausgelösten Erinnerung, sondern vom bewussten Erleben des Fotos. Das Verb przeżyć (>erleben<), przeżyć świadomie (>bewusst erleben $<$ ) drückt die Bedeutung »starke Emotionen erfahren in Verbindung mit bestimmten Ereignissen oder Enttäuschungen $\aleph^{26}$ aus. Ein solches Erleben, das mit positiven wie negativen Geschehen verbunden sein kann, ist tief, stark und offen-

26 Vgl. »Wielki słownik języka polskiego«: https://www.wsjp.pl/ (12.12.2020). 
bart die Sensibilität des Betrachters. Unwichtig werden die Einzelheiten, unbekannt bleibt, wo und wann sich etwas abspielt ${ }^{27}$, weil der liebevolle Erzähler nicht alles sagt. Es bleiben unbestimmbare Stellen der Offenheit, da es sich um das Erleben, um Emotionen, um einen Lebensmoment und nicht um Präzision handelt. Es gibt noch mehr solcher Unklarheiten - die Einzelheiten verlieren sich, sichtbar sind lediglich Umrisse und Schemen, aber über das Sehen werden auch alle anderen Sinne aktiviert. »Das Licht ist weich wie bei Regen, ein Frühjahrslicht ist es wohl [...]« (S. 11), manchmal verstummt der Ton, das Kind hört die Stimme anderer Sonnensysteme und Galaxien zwischen Knacken und Rauschen. (S. 12)

Eigenschaften des liebevollen Erzählers - von Tokarczuk verkörpert und umgesetzt - sind Sensibilität und Aufmerksamkeit. Alles ist für sein Bewusstsein wichtig: alte Gegenstände - das Radio, Knöpfe für die Regulierung der Lautstärke und für die Sendersuche, die empfindlichen Antennenfühler ${ }^{28}$, die Offenheit für die Welt sichern, aber auch der Mensch und die Umstände, in denen sich jemand und etwas befindet, was zur Entdeckung des Inneren, des Seelenzustands, des Bewusstseins einer nahe stehenden Person führt. Die Mutter auf dem alten, undeutlichen Foto ist durch einige Eigenschaften gekennzeichnet: Sie hat ihren Blick auf einen Punkt jenseits des Bildrandes gerichtet, ist leicht gebeugt, wirkt traurig, ist in Gedanken versunken, abwesend. Die Schriftstellerin steht der Mutter sehr nahe, und als eine Person, die das Bild betrachtet, hat sie die Fähigkeit, das mitzuempfinden, was die im Bild >lebende< und fühlende und sehende Mutter empfindet.

So gelingt es der Autorin, eine Erinnerung wachzurufen, wenn sie bei der Beschreibung die Worte sorgfältig wählt und von Gesten, Blicken, Licht spricht; es erlaubt, die Seele zu entdecken, und damit den liebevollsten Erzähler der Welt. ${ }^{29}$ Die Verbindung der Seele ${ }^{30}$ mit dem liebevollen Erzähler unterstreicht die Rolle des Gefühls und der Sensibilität, sie veranlasst auch den/die Rezipient*in dazu, dieses Verstehen der Seele als bevorzugt zu betrachten.

Warum wurde die Fotobeschreibung zum Ausgangspunkt der Festrede, deren Zuhörerschaft so wichtig und zugleich so heterogen ist? Ein Grund dafür ist, dass ein Foto mehrdeutig und vielsagend ist und dass es bei seiner Beschreibung nicht um die Oberfläche geht, sondern um die Entdeckung, was im Foto bzw. was dahinter steckt und was die Vorstellungskraft und Fantasie animiert. Zudem geht es um die Fähigkeit, Interesse zu wecken. So schreibt Susan Sontag zur Rolle der Bilder und zu ihrem Einfluss auf die Weltwahrnehmung:

\footnotetext{
27 Es werden keine Einzelheiten genannt, die Umstände sind lediglich allgemein umrissen. Bekannt ist nur, dass es sich um das Ende der sechziger Jahre und um die westpolnische Provinz handelt; am wichtigsten sind die Erlebnisse des Kindes und das Gespräch mit der Mutter.

28 Im Polnischen gehört das Wort czułki in der Wortgruppe czułki anten als Entsprechung von die empfindlichen Antennenfühler zur Sprachfamilie von czuć, was den Leser für den Sprachgebrauch sensibilisiert.

29 Der von Tokarczuk eingeführte Begriff der Seele wird mit dem von Jung gebrauchten Terminus >anime<, verglichen, verstanden als Exploratorin/Erforscherin des Unbewussten und des Unheimlichen, als Weberin des Schicksals. (Kącka 2020: 7).

30 Hier ist die erste Bedeutungsvariante des Lexems Seele gemeint, also »Gesamtheit dessen, was das Fühlen, Empfinden, Denken eines Menschen ausmacht; Psyche«, vgl. https://www.duden.de/rechtschreibung/ Seele (15.12.2020).
} 
»Jedes Foto ist mehrdeutig: Etwas als Bild zu sehen, heißt einen potenziellen Gegenstand der Faszination zu finden. Die definitive Weisheit eines Fotobildes verbirgt sich hinter der Feststellung: Das ist eine Oberfläche. Und jetzt denkt darüber nach, oder besser erspürt, was dahinter steckt, wie die Wirklichkeit sein muss, wenn sie so aussieht.«(Sontag 2017, S. 31).

\subsection{Die Kategorie die liebvolle Zuneigung}

Im Schlusskapitel (Kapitel 7), also an einer topologisch wichtigen Stelle, wird dagegen die liebevolle Zuneigung an sich charakterisiert. Die darin vermittelten Sachverhalte sind auf die Botschaft der Schriftstellerin zurückzuführen, der ihre Erfahrung als Schriftstellerin sowie ihre Vorstellungskraft zugrunde liegen. Dabei wird die Relevanz der liebevollen Zuneigung hervorgehoben, weil sie durch die positive Hinwendung zu jedwedem Wesen gekennzeichnet ist und die auf dem Gefühl beruhenden zwischenmenschlichen Relationen prägt. ${ }^{31}$ Das Lexem ${ }^{32}$ czułość (>liebevolle Zuneigung $<$ ) erscheint im letzten Kapitel in einer sehr hohen Frequenz. Durch seine Repetition gewinnt der thematisierte Inhalt an Relevanz. Tokarczuk macht deutlich, dass es sich um eine einzige Größe bzw. ein einzigartiges Gefühl handelt, die bzw. das die gespaltene Welt zusammenfügen kann. Man kann der Passage eine geistige, ja religiös-metaphysische Prägung nicht absprechen. Die Botschaft vom liebevollen Erzähler lässt sich folgendermaßen situieren: Im polnischen Original drängt sich eine gewisse Parallele zum Hohelied der Liebe (Hymn o miłości) aus dem 13. Kapitel des 1. Korintherbriefs des Paulus von Tarsus ${ }^{33}$ auf, wobei die eigentliche Beschreibung der Liebe in 13,4-8a erfolgt, von »Die Liebe ist langmütig « bis zu »Die Liebe vergeht niemals « (dazu noch 13,13: »die Liebe ist die größte«).

Die metaphysische Provenienz von Tokarczuks Positionen zu vielen in der Vorlesung behandelten Teilthemen steht außer Zweifel, inwieweit sie aber mit Religion

\footnotetext{
${ }^{31}$ Die Begriffe czułość und czucie (`Zärtlichkeit und Fühlen`) in der polnischen Literatur analysiert Teresa Kostkiewiczowa (2016), indem sie darauf aufmerksam macht, dass sie schon seit der altpolnischen Zeit einem Bedeutungswandel unterlagen und als Begriffe der literarischen Ästhetik fungierten, die dazu dienten, wesentliche Auffassungen zum Schaffensprozess, zur Natur des Werkes und seiner Rezeption auszudrücken. Die Autorin betont den Wandel der erwähnten Begriffe und ihre Abhängigkeit von der jeweiligen Epoche oder Strömung.

32 Im Deutschen eine Wortgruppe.

33 Vgl. https://www.uibk.ac.at/theol/leseraum/bibel/1kor13.htmlgl (10.12.2020).
} 
und mit Gott verbunden sind, müsste aus literaturwissenschaftlicher und philosophischer Perspektive im Hinblick auf ihr Gesamtschaffen behandelt werden. ${ }^{34}$

Aus der charakterisierten Passage könnte man somit im Vergleich mit dem Hohelied der Liebe folgendes zu konstatieren wagen: Die Liebe (miłość) ist die Eigenschaft des Schöpfers, czułość (>Zärtlichkeit $<$ ) soll die des Erzählers werden.

Wenn man die Kohärenz in weiteren Textpassagen verfolgt, ist es wichtig hervorzuheben, dass Tokarczuk durch die Charakterisierung der liebevollen Zuneigung eine Brücke zwischen diesem Gefühl und der Literatur schlägt, wobei die liebevolle Zuneigung als ein immanenter Bestandteil der Literatur angesehen wird:

»Literatur gründet auf der liebevollen Zuneigung, die wir jedem anderen Sein entgegenbringen. Und das ist der entscheidende psychologische Mechanismus des Romans. Dem wunderbaren Instrument der liebevollen Zuneigung - der raffiniertesten Art der menschlichen Kommunikation - ist es zu verdanken, dass unsere Erfahrung durch die Zeit reisen und jene erreichen kann, die noch nicht geboren sind, aber einmal das zur Hand nehmen werden, was wir über uns und unsere Welt erzählt haben.«(S. $60 \mathrm{f}$.)

Ergänzend ist hinzuzufügen, dass die Autorin die liebevolle Zuneigung mit der Fiktion, die der Literatur eigen ist, in Verbindung setzt. Und zwar findet sich zur Fiktion bei Tokarczuk die folgende Bestimmung:

»Ich schreibe fiktionale Geschichten, doch sind diese Geschichten niemals gänzlich »erfunden«. Wenn ich schreibe, muss ich alles in mir fühlen. Der Weg aller Wesen und Dinge in meinen Büchern, alles Menschlichen und AuBer-Menschlichen, alles Lebendigen und Nicht-mit-Leben Beschenkten führt durch mich. Jedes Ding und jede Person betrachte ich lange und aufmerksam, um sie zu verkörpern, zu personifizieren.

Dazu dient mir mein liebevoller Blick - denn liebevoll zu sein, ist die Kunst der Verkörperung, der Einfühlung, der fortwährenden Suche nach Gemeinsamkeiten.«(S. 58)

In Anschluss an die voranstehende Analyse seien noch Kontexte genannt, in denen von der liebevollen Zuneigung im Sinne einer Implizität gesprochen werden

\footnotetext{
${ }^{34}$ Das Lexem czułość (>liebevolle Zuneigung ) kommt im Original als Anapher in drei von vier Absätzen vor. Im zweiten Absatz wird es in substantivischer Form nicht verwendet, das Personalpronomen ona ( $>$ sie $<$ ) bleibt als Wiederaufnahme ebenfalls ausgespart. Interessanterweise wird der dritte Absatz des Originals in der Übersetzung durch zwei einzelne Absätze wiedergegeben. Es ist hier hinzuzufügen, dass im Polnischen Personalpronomina als Wiederaufnahmen meistens ausgelassen und stattdessen nur bei der Hervorhebung verwendet werden.

So könnte diese einer Litanei ähnelnde Aufzählung - gemeint ist hier das Original - als eine weltliche, jedoch zugleich auch eine geistig geprägte Entsprechung dieses Hymnus fungieren, in der czułość in den Mittelpunkt rückt. Dazu trägt ebenfalls ein durch einzelne Absätze gebildeter Rhythmus bei.

In der Übersetzung wird dagegen diese Aufzählung nicht übernommen: So fehlt die Repetition, zwei Absätze enthalten nur die Wortgruppe »Die liebevolle Zuneigung « (Abs. 1) und »Liebevolle Zuneigung « (mit dem Nullartikel) (Abs. 3) (S. 59), die zwei weiteren werden mit anderen sprachlichen Mitteln wiedergegeben: »Der liebevolle Blick« und »In der zugeneigten Betrachtung« (S. 60).
} 
kann, d. h. dass die Formulierung nicht explizit verwendet wird, sondern dass sie vielmehr der poetischen Struktur immanent ist. ${ }^{35}$

Die liebevolle Zuneigung manifestiert sich auch in der Haltung der Autorin. Es wird gezeigt, dass das Interesse der Schriftstellerin allen Lebewesen und Dingen der Welt gilt - Tokarczuk spricht von einem anderen Sein im Allgemeinen (ebenda, S. 59) - die Welt der Lebewesen wird als Aufgabe des Menschen dargestellt, sich gleichsam zu jedem Sein und Seienden niederzubeugen. (Vgl. die voranstehende Passage in diesem Kapitel, die die Fiktion thematisiert.) Dies kann als Tokarczuks Credo bezeichnet werden.

Auch bei der Charakterisierung der liebevollen Zuneigung wird die Welt der Anderen in ihrer Existenz thematisiert: Die liebevolle Zuneigung erscheint überall, »Wo wir unseren Blick eingehend und behutsam auf ein anders Sein richten, auf etwas, das nicht >Ich< ist.« (ebenda, S. 59)

Eine solche Haltung, d. h. die Betrachtung eines anderen Seins, »geht weit über empathisches Mitfühlen hinaus.« (ebenda). Die Autorin macht in einigen Passagen deutlich, dass ihr Sensibilität gegenüber den Dingen viel bedeutet, auch die Personifizierung der Sachen spielt im Text eine wichtige Rolle, wie bei der Anknüpfung an ein Märchen von Andersen, in dem sich die Teekanne beklagt, »sie sei von den Menschen grausam behandelt worden« (S. 40), Dinge erzählten Geschichten, Teller unterhielten sich miteinander (S. 40f.) Eine spirituelle Verbindung mit der Welt (mit Pflanzen, Tieren, Dingen), der sich die Autorin in ihrer Kindheit bewusst war, führte sie somit zu ihrer späteren Lebenshaltung.

Es ist kein Zufall, dass Tokarczuk nach Metaphern greift, deren neue Rolle in der modernen Forschung entdeckt und in der kognitiven Linguistik besonders hervorgehoben wird. Sie setzen uns »in grundsätzlicher Weise Erkenntnis-Brillen auf « ${ }^{36}$. Metaphern dienen der Autorin beispielsweise dazu, die liebevolle Zuneigung bildhaft darzustellen und dem Prozess des Geschichtenerzählens lebendige Plastizität $\mathrm{zu}$ verleihen. ${ }^{37}$ Wie bereits betont, gelangen die Metapher des Webens und einige isotope Bezeichnungen zur Anwendung: »Die Welt ist ein Stoff, an dem wir täglich weben - auf großen Webstühlen verarbeiten wir Fäden (...).« (Tokarczuk 2020c, S. 15), was nicht zufällig mit der Bezeichnung >Text $<$ verbunden wird, in dem wohl nicht selten eine Geschichte vermittelt wird, der ursprünglich, nämlich etymologisch, eben Gewebe bedeutete ${ }^{38}$. Die Wahl einer solchen bildhaften Metapher ist also keinesfalls zufällig oder beliebig.

\footnotetext{
35 Man kann sich Kozłowska (2020, S. 1) anschließen, wenn sie postuliert, dass sich die liebevolle Zuneigung im Können manifestiert, kleine Dinge in der Atmosphäre wahrzunehmen und sie in ein Gespräch über unsere Welt einzubeziehen, was zum ehrlichen und kritischen Denken führt. Dies mache übrigens der Autorin zufolge den Unterschied zur Propagandasprache aus.

$36 \mathrm{Vgl}$. https://sprachkompass.ch/theorie/metaphern-erkennen (18.12.2020).

37 In der umfangreichen Literatur zur Metapher werden ihre Funktionen in der Literatur, aber auch in Fachtexten und in der Alltagssprache erforscht und u. a. ihre Bildhaftigkeit hervorgehoben, aber auch darauf hingewiesen, dass Metaphern unser Denken formen, vgl. dazu u. a., aber insb. Blumenberg (1999), Weinrich (1972), Haverkamp (1983), aber auch die Arbeiten von amerikanischen Vertreter der kognitiven Linguistik, u. a. Lakoff, Johnson (1980) und Übersetzung ins Deutsche: Lakoff und Johnson (2018). In der polonistischen Forschung vgl. Dobrzyńska (1984).

38 Vgl. Text (...) eigentlich >Gewebe, Geflecht<, einer Bildung zu lat. texere (textum) >weben, flechten, zusammenfügend verfertigen, bauen, errichten< (...): https://www.dwds.de/wb/texten (15.12.2020).
} 
Die liebvolle Zuneigung verursacht auch, dass ein einfaches Ding magisch wird, was die folgende Personifizierung deutlich macht: »Liebevolle Zuneigung bringt die Teekanne zum Sprechen.«(S. 59) Die kindliche Sensibilität als natürlicher Zug des Kindes und sein Glaube daran, dass nicht nur Tiere und Pflanzen Lebewesen und spirituell verbunden sind, sondern auch die Landschaft mit all ihren Bestandteilen, ja sogar Dinge sind durch ihre eigene Existenz gekennzeichnet. (vgl. Kapitel 4) Diese kindliche Betrachtung ist für Tokarczuk ein Zustand, eine Weise des Seins, nach der sie sich sehnt. Die zeitgenössische Welt, die wir fragmentarisch und in Bruchstücken sehen, soll, wie es im folgenden Text steht, mit Hilfe der liebevollen Zuneigung als Ganzheit erzählt werden oder noch grundsätzlicher: überhaupt erzählbar werden.

Dank der liebevollen Zuneigung werden auch Bande, Gemeinsamkeiten und Übereinstimmungen zwischen Menschen entdeckt: »Sie zeigt die Welt als lebend und lebendig, als ineinander verbunden, voneinander abhängend, zusammenwirkend.« (S. 60)

Den Bedrohungen in der gegenwärtigen Welt, der Zerbrechlichkeit der menschlichen Angelegenheiten stellt Tokarczuk die liebevolle Zuneigung gegenüber, wodurch ein Kontrast konstituiert wird.

\section{Das Konzept czuły narrator (>der liebevolle Erzähler $<$ ) - scheinbare Widersprüche?}

Wir haben uns u. a. auf die für das Textganze konstitutiven Analogien eingelassen (der liebevolle Erzähler als Seele, als eine Instanz mit einer äußerst positiven Wertung), die sich bei dem liebevollen Erzähler bemerkbar machen. Im Anschluss sollen dagegen scheinbare Widersprüche offengelegt werden, die mit dieser Schlüsselinstanz zusammenhängen. Es ist dabei auf die >Beschaffenheit< des liebevollen Erzählers als Konzept, auf seine Rolle in der Literatur sowie auf seine Beziehung zur Welt einzugehen.

Dem/der Leser*in kann, wie bereits angemerkt, eine pragmatische Offenheit beim Deuten der Schlüsselinstanz nicht abgesprochen werden. Der Bezug auf den IchErzähler und die Thematisierung der uns umgebenden Welt mit ihren Problemen und Bedrohungen stellt jedoch einen Rahmen dar, der die Interpretation im gewissen Sinne steuert. Als wichtig für die Gesamtinterpretation ist jedoch die Verschränkung der fundamentalen Größen (Erzähler, Erzählung, literarisches Werk und die Welt), wobei von besonderem Interesse die Schnittstellen zwischen diesen Größen sind.

Die Schlüsselinstanz - der liebevolle Erzähler als neues literarisches Konzept, jedoch nicht als eine Kategorie der Erzähltheorie -, wird als eine mysteriöse Gestalt konzipiert und inszeniert, die »die Perspektive sämtlicher Figuren mit einnimmt und zugleich den Horizont jeder einzelnen überschreitet, der mehr und weiter sucht, der die Zeit außer Acht lassen kann. O ja, ein solcher Erzähler ist möglich.« (S. 52) 
Damit wird der liebevolle Erzähler, anders gesagt, der Erzähler in der vierten Person ein Novum ${ }^{39}$, in dem Sinne, dass ihn Tokarczuk zum ersten Mal prägt und charakterisiert. Auch in der Narrationstheorie gelangt ein solcher Terminus nicht zur Verwendung. Als entscheidend erweist sich hierbei die Überzeugung Tokarczuks, der zufolge es eine geistige Kraft gibt, die die Sachlage in einem Bereich beeinflussen oder sogar verändern kann (vgl. die Szene mit der Mutter). Im Kontext der früheren Ausführungen der Nobelpreisträgerin sorgt folglich die Konstatierung »Und Vorstellbarkeit ist schließlich die erste Stufe des Seins.« (Schlusssatz im Kapitel 6, S. 58) ebenso wenig für Zweifel, die geistige Kraft bewahrt auch hier ihre Gültigkeit. Die Vorstellungskraft ist es eben, die den Ausgangspunkt zur Veränderung alles Bestehenden darstellt, und noch mehr: Der liebevolle Erzähler und die geistige Kraft, die ihm eignet bzw. die ihn hervorbringt, ist nicht nur in der Literatur angesiedelt, dieser Erzähler wird auch mit der realen Welt in Verbindung gesetzt. Dieser Erzähler ist derjenige, der als Urheber eines galaktischen Wandels gelten könnte. Fraglos ist es der Akt der schriftstellerischen Inspiration, die den liebevollen, d. h. empathischen, gefühlsbetonten und aufmerksamen Erzähler hervorbringt. Seine Wirksamkeit besteht in seinen Attributen, die ihn als äußerst positiv kennzeichnen. Im Hinblick auf die uns umgebende Welt, die so viele existentielle Mängel und Nöte hat und Besorgnis erregt, kann jedoch diese Wirksamkeit auch zugleich in Frage gestellt werden. Dieser Gedanke wird zwar nicht explizit formuliert, aber die identifizierten Probleme der Welt stehen im deutlichen Widerspruch zu den Eigenschaften und Handlungsweisen des wunderbaren Erzählers, mit seiner Milde und Sensibilität, so dass zwei verschiedene Sphären, die der Welt und die der Literatur, aufeinander prallen. Tokarczuks Stellung zur literarischen Fiktion, die sie als eine Art Wahrheit im Hinblick auf die Kategorie > Literatur < bezeichnet, wird mit einer negativen Einschätzung des Verhältnisses von Fiktion und Welt korreliert und mit der in dieser Welt-der-Nöte verbreiteten Lüge konfrontiert. Die beiden Sphären (die Welt der Literatur und die der uns umgebenden Welt) werden zueinander in Beziehung gesetzt, wobei hier die Grund-Folge-Beziehung markiert wird:

»Seit die Lüge zu einer - wenngleich immer noch reichlich primitiven - Massenvernichtungswaffe geworden ist, hat sich das Vertrauen der Leser in die Fiktion verflüchtigt. Immer häufiger stellt man mir die Frage, und Ungläubigkeit schwingt darin mit: »Ist es denn wahr, was Sie da geschrieben haben? « Und jedes Mal habe ich das Gefühl, das Ende der Literatur sei nahe - klingt doch diese in der Wahrnehmung der Leser harmlose Frage für schriftstellerische Ohren wahrhaft apokalyptisch. Was soll ich darauf antworten? Wie lässt sich der ontologische Status eines Hans Castorp, einer Anna Karenina oder eines Pu der Bär erklären?«(S. 30f.)

\footnotetext{
39 Von diesem Erzähler schreibt Tokarczuk (2020e) ebenfalls in dem bereits erwähnten Essay Psychologia narratora; wie angemerkt, wird er dort als narrator czwartoosobowy bezeichnet, wobei in dem hier analysierten Text die Bezeichnung czuly narrator zur Verwendung gelangt, narrator czwartoosobowy dagegen als sein Synonym fungiert und im Text nur einmal (Kapitel 6) erscheint. Es ist somit deutlich zu sehen, dass die Autorin die erste Bezeichnung bevorzugt.
} 
Von Bedeutung erscheint hier die Frage, wie Tokarczuk den Zustand der Welt als Folge des vielfach desaströsen Handelns des Menschen mit der Literatur und deren Aufgaben in Verbindung setzt. Fest steht, dass der Schriftstellerin die Probleme der heutigen Welt sehr wichtig sind, darunter besonders die der Umwelt. Die Überzeugung, dass Natur und Mensch eine unzertrennliche Einheit bilden und dass der Mensch (wie auch andere Lebewesen und ebenso Dinge) ein immanenter Teil der Natur sind, findet man in vielen Werken der Autorin. In dem hier im Mittelpunkt stehenden Text manifestiert sich diese Sorge deutlich in folgenden Worten: »Uns alle - Menschen, Pflanzen, Tiere, Dinge - umschließt ein und derselbe, von physikalischen Gesetzen regierte Raum. Dieser gemeinsame Raum besitzt seine eigene Gestalt, innerhalb derer die physikalischen Gesetze eine unzählige Menge aufeinander bezogener Formen herausbilden.« (S. 50)

Der liebvolle Erzähler - unzertrennlich verbunden mit der Literatur und Fiktion ebenso wie mit der Welt da draußen - wird als eine komplexe, mehrdimensionale Größe postuliert, was seine Deutung zwar nicht erleichtert, jedoch seine Zuständigkeiten ordnet.

Wer ist nun eigentlich der liebevolle Erzähler? Er bleibt im Laufe der dargestellten Ausführungen im gewissen Sinne mysteriös, ja, er scheint diese mysteriöse Unschärfe behalten zu müssen, obwohl er im Kontext des Gesamtvortrags doch bestimmte Konturen annimmt.

Da, wo sich Tokarczuk zum Zustand der Welt äußert, erscheint sie nicht als reine Kritikerin der existierenden Lage; sie versucht auch ein Heilmittel zu finden, und als Schriftstellerin ist dieses für sie der liebevolle Erzähler. Es besteht kein Zweifel darüber, dass sie die beiden Welten, die der Wirklichkeit und die der Literatur, mit dem liebevollen Erzähler in Beziehung setzt. Wie ist also die Haltung der Nobelpreisträgerin zu deuten, wenn sie Fragen der Fiktion, das Plädoyer für eine ganzheitliche Betrachtung der Welt, den Mangel an einem holistischen Denken im Hinblick auf die Relation Mensch - Natur und schließlich die systematische Vernichtung der Welt thematisiert?

Die negativen Erscheinungen in der heutigen Welt werden eindeutig auf das zerstörerische Handeln des Menschen zurückgeführt: »Häufig vergessen wir, dass $\mathrm{sie}^{40}$ kein Fatum, kein Schicksalsschlag ist, sondern das Ergebnis ganz konkreter Schritte und wirtschaftlicher, gesellschaftlicher, weltanschaulicher (auch religiöser) Entscheidungen.«(S. 61)

Die Darstellung der Zivilisationsprobleme ${ }^{41}$ wird mit den Konzepten der Literatur in Beziehung gesetzt, insbesondere mit der Erzählinstanz, was auf den ersten Blick als unverständlich erscheinen kann. Aber bei dem postulierten liebevollen Erzähler ist eben das Geschichteweben sein Attribut, d. h. die Art und Weise über die Welt so zu erzählen, dass sie als holistisch verstanden wird. Tokarczuk sieht eine sehr enge Beziehung zwischen der Literatur und der Welt in Verbindung mit ihrer eigenen Aufgabe als Schriftstellerin:

\footnotetext{
40 Gemeint ist die klimatische und politische Krise (S. 61).

41 Die engagierte Haltung Olga Tokarczuks den politischen und sozialen Problemen in Polen gegenüber ist bekannt.
} 
»Gier, mangelnde Achtung vor der Natur, Egoismus, Phantasielosigkeit, ein nicht enden wollender Wettstreit haben die Natur zu einem Ding gemacht, zu einem Objekt, das man in Stücke schneiden, benutzen und zerstören kann.

Und deswegen glaube ich fest daran, dass ich so erzählen muss, als wäre die Welt eine lebendige, vor unseren Augen immerfort im Werden begriffene Einheit - und wir ein kleiner und zugleich mächtiger Teil dieser Welt.« (S. 62)

Tokarczuk macht deutlich, dass diejenigen Erscheinungen und Einstellungen, die sich heute im Allgemeinen keines großen Interesses erfreuen, d. h. die Fiktion, Anknüpfungen an den Mythos, eine holistische Auffassung von der Welt, vor allem aber die liebvolle Zuneigung gegenüber allem Seienden nicht nur für die Literatur von Bedeutung sind, sondern dass sie die Macht besitzen, als geistige Größen ${ }^{42}$ und Orientierungshilfen ihre Wirksamkeit in der Welt zu zeigen. Wie das Realität werden kann, mag Tokarczuks letzter, bereits oben angeführter Satz aus der Perspektive ihrer Aufgabe als Schriftstellerin beantworten: Die zersplitterte Welt, so die Schriftstellerin, sei so darzustellen, als ob sie eine Einheit bilden würde. ${ }^{43}$

Wichtig ist hier die Konjunktion deswegen, die als Verknüpfungsmittel mit dem voranstehenden Satz fungiert und eine Grund-Folge-Beziehung markiert. Die Kohärenz mit dem Satz davor besteht darin, eine Relation zwischen der zerstörerischen Tätigkeit des Menschen und der Reaktion der Schriftstellerin darauf zu stiften. Dem Erzähler und dem Erzählen wird dadurch die wirksame Kraft bestätigt, die Welt im positiven Sinne beeinflussen zu können. Das Erzählen ist jedoch ein Prozess der Kreation, der als Aufgabe eines Schriftstellers/einer Schriftstellerin gilt. Wie kann jedoch die Beziehung zwischen der, wie Tokarczuk (2020c) an anderer Stelle schreibt, im Sterben liegenden Welt und dem liebevollen Erzähler gedeutet werden? Zwei Passagen mögen eine Antwort aufzeigen, in denen nicht zufällig der Ausdruck im Sterben liegen in Bezug auf die Kondition der Welt und das Verb vergehen ${ }^{44}$ in Bezug auf das Nicht-Erzählte zur Verwendung gelangen:

»Die Welt liegt im Sterben ${ }^{45}$, doch nicht einmal das bemerken wir. Wir bemerken nicht, dass sie allmählich zu einem Sammelsurium von Dingen und Ereignissen wird, zu einem leblosen Raum, in dem wir einsam und verloren herumstolpern, hin- und hergeworfen von Entscheidungen, die nicht wir selbst treffen, Sklaven eines unverständlichen Fatums, verfolgt von dem Gefühl, Spielball der Geschichte oder des Zufalls zu sein.« (S. 43)

»Wie wir über die Welt denken und - vermutlich noch wichtiger - wie wir von ihr erzählen, hat daher eine ungeheure Bedeutung. Was geschieht, aber nicht erzählt wird, hört auf zu sein und vergeht. [...] Wer an der Geschichte webt, hat die Macht.« (S. 15) (S. 62)

\footnotetext{
42 Vgl. die voranstehenden Ausführungen zum liebevollen Erzähler.

43 Vgl. das voranstehende Zitat.

44 Interessanterweise wird im Polnischen das Verb umierać (>sterben<) an den beiden erwähnten Stellen gebraucht. Im zweiten Fall handelt es sich um eine Personifizierung, die im polnischen Text eine stärkere Wirkung als der Ausdruck in der übersetzten Version zeigt.

45 Hervorhebung in den beiden Zitaten - Z.B.-H., M.K.
} 
Das Erzählen kann somit das noch-nicht-Existierende erschaffen, so wie das bereits mehrmals thematisierte Gefühl des Vermissens von etwas-noch-nicht-Existierendem das, was also noch nicht existiert ins Leben rufen, es erwecken, ja verlebendigen oder auch bewahren kann. Mit einer solchen Deutung ist der scheinbare Widerspruch zwischen der Kondition der heutigen Welt und dem liebevollen Erzähler als Repräsentanten der Literatur teilweise aufgehoben. Könnten seine Sensibilität, sein Einfühlungsvermögen und seine holistisch geprägte Vorstellungskraft ein wirksames Mittel gegen die sterbende Welt sein, nicht nur im materiellen, sondern auch im geistigen Sinne? Oder zugespitzt ausgedrückt: Sind sie vielleicht das einzige Remedium überhaupt?

Die Worte der Nobelpreisträgerin können so gedeutet werden: Der liebevolle Erzähler soll über die Welt so erzählen, dass er imstande ist, den/die Leser*in zu bewegen, den Problemen der Welt gegenüber nicht gleichgültig zu bleiben, seine liebevolle Zuneigung in sich zu aktivieren und weiterzuentwickeln. Die Rolle des/der Leser*in sollte somit in der bewussten Wahrnehmung der negativen Erscheinungen der Welt bestehen, was eine aktive, kritische Haltung auslösen sollte, sich mit ihnen auseinanderzusetzen und gegen sie anzukämpfen.

Schließlich ist noch auf den von Tokarczuk präsentierten holistischen Gedanken aufmerksam zu machen, der beim Erzählen als Prozess eine fundamentale Rolle spielt.

»Und daraus folgt, dass es offen und ehrlich zu erzählen gilt, so, dass die Geschichten im Geist des Lesers einen Sinn für die Ganzheit anregen, dass sie die Gabe in ihm wecken, Bruchstücke zu einem Muster zu vereinen und in kleinsten Ereignissen die Konstellationen des Ganzen zu erkennen. Geschichten gilt es zu spinnen, in denen deutlich wird, dass alle und alles in einer gemeinsamen Vorstellung eingeschlossen sind, die wir mit jeder Drehung unseres Planeten in unserem Geist erzeugen.« (S. 54)

Damit wird eine geistige Bindung zwischen den Menschen postuliert und eine Art der Gemeinschaft, aber auch, wie die Autorin fortsetzt, die Gleichwertigkeit der Rolle von Autor*innen und Leser*innen in ihrem in der Welt-Sein betont. Denn das Erzählen wird bei Tokarczuk breit verstanden und beschränkt sich nicht nur auf den Schaffensprozess. ${ }^{46}$

Dem Konzept des liebevollen Erzählens steht ein entsprechender Gedanke von Jerzy Trzebiński (2002) nahe, der schreibt, dass die Narration die Art und Weise ist, die Welt zu verstehen und dass man mit ihrer Hilfe die Welt konstruieren kann. Olga Tokarczuks Erzähler vereint in sich Eigenschaften des Erzähler-Schriftstellers und des Erzähler-Menschen, der eine Geschichte über die gegenwärtige Welt spinnt, über die Bedrohungen, die uns alle betreffen, und der Antworten auf Fragen sucht, wie man die Wirklichkeit erfassen soll (schreibend, erzählend, sprechend, kommentierend, eine Geschichte/Geschichten konstruierend), um »eine solch gewaltige konstellative Form der Welt zu stemmen [...]« (Tokarczuk 2020c, S. 45).

46 Dies ist jedoch ein Thema, das gesondert behandelt werden sollte. 


\section{Schlussbemerkungen}

Bei der Interpretation des Konzepts des liebevollen Erzählers ist resümierend zu bemerken, dass diese Figur auf mehreren Bezugsebenen angesiedelt ist. Auf einer ersten Ebene handelt es sich um eine innovative Kategorie, die von Tokarczuk postuliert wird. Er ist ein Erzähler in der vierten Person, der dem Ich-Erzähler gegenübergestellt und mit neuen Eigenschaften ausgestattet wird.

Tokarczuk bestimmt den liebevollen Erzähler als jemanden, der »die Perspektiven sämtlicher Figuren mit einnimmt und zugleich den Horizont jeder einzelnen überschreitet, der mehr und weiter sieht, der die Zeit außer Acht lassen kann.« (S. 52) In diesem Sinne zeigt er auch Ähnlichkeiten zum auktorialen Erzähler, jedoch besitzt er noch andere Eigenschaften, von denen in der Analyse die Rede war: Er verbindet verschiedene Gesichtspunkte, Empfindungen und Perspektiven zu einem kohärenten Ganzen.

Auf einer zweiten Ebene drückt die Schriftstellerin ihre Überzeugung aus, dass ein solcher Erzähler auch als >Weltfigur < möglich ist. Er wird damit als Konzept postuliert, aber auch zugleich in dem hier analysierten Text im praktischen Sinne als >Methode $<$ umgesetzt, indem die Autorin implizit die liebvolle Zuneigung im Prozess des Konstruierens ihres Textes verdeutlicht. Diese zweite Ebene ist im Bereich der schriftstellerischen Arbeit angesiedelt. Während aber das Konzept ganzheitlich ausgerichtet ist, können in dem Text der Vorlesung nur einige Aspekte durch ausgewählte Schritte und Strategien präsentiert werden. Die liebevolle Zuneigung als Eigenschaft des neuen Erzählers kann in diesem Sinne als eine >Theorie < des Geschichtewebens betrachtet werden. Die Autorin gebraucht zwar grundlegende Termini der Erzähltheorie, verwendet aber eine bildhafte Sprache, um die Vorstellungskraft des/der Adressat*in anzuregen. Er kann die Welt dadurch ansprechen, dass er ein Bedürfnis hat, eine Geschichte immer aufs Neue zu erzählen, und zwar in verschiedenen Schattierungen und Varianten. Tokarczuk wird als Schriftstellerin selbst zur >Weberin< einer Geschichte (vgl. die Metapher des Webens). Die Schriftstellerin plädiert für eine »neue Universal - Erzählung [...], für eine ganzheitliche, allumfassende, in der Natur verwurzelte Narration, die die unterschiedlichsten Kontexte mit einbezieht und dennoch verständlich bleibt.« (S. 51)

Diese Narration betrachtet die Nobelpreisträgerin als einen Prozess, und sie stellt die Frage nach der Art und Weise seiner Verwirklichung: »Wie soll man schreiben, wie eine Geschichte konstruieren, die in der Lage wäre, eine solch gewaltige konstellative Form der Welt zu stemmen? (S. 45) Diese Aufgabe erscheint als besonders wichtig, weil sie als eine Art >Heilmittel < gegenüber den Problemen der Welt betrachtet werden kann - womit die dritte Ebene betreten wäre, die auf der Achse der liebevolle Erzähler (mit seinem Geschichtenerzählen) - Leser - Welt angesiedelt ist.

In Tokarczuks Text handelt es ich um ein literarisches Konzept des zärtlichen Erzählers, das u. a. dazu dient, zwischen der Literatur, dem Leser und der Welt eine Brücke zu schlagen, aber auch noch um etwas mehr zu erreichen. Fokussiert wird auf die Lage der Welt, die durch das destruktive Handeln der Menschen bedroht ist. Die Vorlesung ist somit nicht nur als eine Diagnose der Gegenwart zu verstehen, sondern als explizites Plädoyer, umzudenken und auch beim Handeln die Welt als 
eine Ganzheit von Lebewesen und Dingen zu begreifen. Dass sich dies auf der Achse der Literatur - Leser - Welt abspielt, darf im Falle einer Schriftstellerin nicht verwundern. Das Erzählen (das Geschichteweben) könnte hier als ein Postulat der Erziehung des Menschen zum Umdenken und Umwerten verstanden werden. Es suggeriert einen notwendigen Prozess, der bald starten und systematisch entwickelt werden solle, weil die Welt es so dringend brauche. Genau so lässt sich der an der Universität Łódź gehaltene und später publizierte Vortrag Tokarczuks »Psychologia narratora « (〉Psychologie des Erzählers $<$ ) verstehen, der eine durchdachte Konzeption des Erzählers bietet und den sie mit folgenden Worten schließt: »Der Mensch, meine Damen und Herren, meine Lieben, hat Seele, Körper und Erzähler.« (2020e, S. 179). ${ }^{47}$

Der liebevolle Erzähler kann somit als ein komplexes Konzept verstanden werden, das uneindeutig bleibt: trotz vieler Eigenschaften, die ihm zugeschrieben wurden; das, um es noch anders auszudrücken, als offene Form anzusprechen ist. Dieser Erzähler ist, wie deutlich werden sollte, nicht mit den Modellen der Erzähltheorie und den Typologien des Erzählers zu erfassen, er ist, was sich aus den Ausführungen Tokarczuks ergibt, eine Leistung und Kreation der Schriftstellerin. Er ist einerseits eng mit ihrer Intuition, Vorstellungskraft und ihrem Talent als Schriftstellerin verbunden, andererseits erwächst er aus ihrer subjektiven Betrachtung des Schaffensprozesses und ist zugleich eine Instanz, wenn nicht Autorität, deren durch Gefühl geprägte Haltung den Menschen im positiven Sinne beeinflussen könnte.

Funding Open access funding provided by University of Rzeszow.

Open Access Dieser Artikel wird unter der Creative Commons Namensnennung 4.0 International Lizenz veröffentlicht, welche die Nutzung, Vervielfältigung, Bearbeitung, Verbreitung und Wiedergabe in jeglichem Medium und Format erlaubt, sofern Sie den/die ursprünglichen Autor(en) und die Quelle ordnungsgemäß nennen, einen Link zur Creative Commons Lizenz beifügen und angeben, ob Änderungen vorgenommen wurden.

Die in diesem Artikel enthaltenen Bilder und sonstiges Drittmaterial unterliegen ebenfalls der genannten Creative Commons Lizenz, sofern sich aus der Abbildungslegende nichts anderes ergibt. Sofern das betreffende Material nicht unter der genannten Creative Commons Lizenz steht und die betreffende Handlung nicht nach gesetzlichen Vorschriften erlaubt ist, ist für die oben aufgeführten Weiterverwendungen des Materials die Einwilligung des jeweiligen Rechteinhabers einzuholen.

Weitere Details zur Lizenz entnehmen Sie bitte der Lizenzinformation auf http://creativecommons.org/ licenses/by/4.0/deed.de.

\section{Literatur}

\section{Verwendete Literatur}

Adamzik, Kirsten (2001): Die Zukunft der Textsortenlinguistik. Textsortennetze, Textsortenfelder, Textsorten im Verbund. In: Ulla Fix/Stephan Habscheid/Josef Klein (Hg.): Zur Kulturspezifik von Textsorten. Tübingen: Stauffenberg, S. 15-30.

\footnotetext{
47 In der Nobelpreisvorlesung sind intertextuelle Bezüge auf diesen Essay zu finden, die hier jedoch nicht unter die Lupe genommen werden konnten.
} 
Awdiejew, Aleksy (2007): Gramatyka interakcji werbalnej. Kraków: Wydawnictwo Uniwersytetu Jagiellońskiego.

Awdiejew, Aleksy, Habrajska, Grażyna (2006): Typologia emotywnych aktów mowy. In: Kazimierz Michalewski (Hg.): Wyrażanie emocji. Łódź: Wydawnictwo Uniwersytetu Łódzkiego, S. 9-12.

Bańko, Mirosław (2008): Wielki słownik wyrazów bliskoznacznych. Warszawa: Wydawnictwo Naukowe PWN.

Bilut-Homplewicz, Zofia (2013): Prinzip Perspektivierung Germanistische und polonistische Textlinguistik - Entwicklungen, Probleme, Desiderata. Teil I: Germanistische Textlinguistik. Frankfurt a. M.: Peter Lang Verlag.

Blumenberg, Hans (1999): Paradigmen zu einer Metaphorologie. Frankfurt a. M.: Suhrkamp Verlag.

Dijk van, Teun (1985): Działanie, opis działania a narracja. In: Pamiętnik Literacki 1, S. 145-166.

Długosz-Kurczabowa, Krystyna (2006): Stownik etymologiczny jezyka polskiego. Warszawa: Wydawnictwo Naukowe PWN.

Dobrzyńska, Teresa (1974): Delimitacja tekstu literackiego. Wrocław: Zakład Narodowy im. Ossolińskich.

Dobrzyńska, Teresa (1984): Metafora. Wrocław/Warszawa/Kraków/Gdańsk/Łódź: Wydawnictwo Polskiej Akademii Nauk.

Dobrzyńska, Teresa (1993): Tekst. Próba syntezy. Warszawa: Instytut Badań Literackich.

Filip, Grażyna/Krauz, Maria (2010): Szkice o jezzyku $i$ wartościach w wybranych utworach Stefana Żeromskiego. Rzeszów: Wydawnictwo Uniwersytetu Rzeszowskiego.

Genette, Gérard (2010): Die Erzählung. Paderborn: Wilhelm Fink. 3., durchgesehene und korrigierte Auflage übersetzt von Andreas Knop mit einem Nachwort von Jochen Vogt.

Grochala, Beata (2013): Komentarz sportowy jako narracja. In: Dorota Filar/Dorota Piekarczyk (Hg.): Narracyjność jezyka i kultury. Literatura i media. Lublin: Wydawnictwo UMCS, S. 237-246.

Haverkamp, Anselm (1983): Theorie der Metapher. Darmstadt: Wissenschaftliche Buchgesellschaft.

Kajtoch, Wojciech (2021): Wykład wykładu. Rzecz o mowie noblowskiej Olgi Tokarczuk / The Lecture about a Lecture. On Olga Tokarczuk's Nobel Speech. Kraków: Wydawnictwo Uniwersytetu Jagiellońskiego.

Kantner, Katarzyna (2019): Jak działać za pomoca stów? Proza Olgi Tokarczuk jako dyskurs krytyczny. Kraków: Universitas.

Kącka, Eliza (2020): Dar bogini. In: Znak 779, S. 6-13.

Kostkiewiczowa, Teresa (2016): Czucie (czułość, uczucie, tkliwość). In: Józef Bachórz/Grażyna Borkowska/Teresa Kostkiewiczowa/Magdalena Rudkowska/Mirosław Strzyżewski: Stownik polskiej krytyki literackiej. 1764-1918. T.1. Pojecia - terminy - zjawiska-przekroje. Toruń: Wydawnictwo Naukowe Uniwersytetu Mikołaja Kopernika, S. 136-143.

Kozłowska, Dominika (2020): Czułość i odwaga. In: Znak 779, S. 1.

Kulas, Piotr (2014): Narracja jako przedmiot badań oraz kategoria teoretyczna w naukach społecznych. In: Kultura i Spoteczeństwo 4, S. 111-130.

Lakoff, George/Johnson, Mark (1980): Metaphors we live by. Chicago: University of Chicago Press.

Lakoff, George/Johnson, Mark (2018): Leben in Metaphern. Konstruktion und Gebrauch von Sprachbildern. Heidelberg: Carl-Auer Verlag, 9. Auflage.

Markiewicz, Henryk (1984): Wymiary dzieła literackiego. Kraków/Wrocław: Wydawnictwo Literackie.

Martinez, Matias/Scheffel, Michael (2016): Einführung in die Erzähltheorie. München: Beck 2016, 10. überarbeitete und aktualisierte Auflage.

Nalepa, Marek (2013): Demony, duchy i potworki, czyli kłopoty z dorastanie. In: (Hg.): Magdalena Rabizo-Birek/Magdalena Pocałuń-Dydycz/Adam Bienias: Światy Olgi Tokarczuk. Rzeszów: Biblioteka Frazy. Wydawnictwo Uniwersytetu Rzeszowskiego, S. 123-133.

Sontag, Susan (2017): O fotografii. Kraków: Wydawnictwo Karakter, übersetzt von S. Magala.

Stanzel, Franz K. (1993): Historia komplementarna. Zarys zwróconej ku czytelnikowi teorii powieści. In: Pamietnik Literacki 1, S. 205-222.

Stanzel, Franz K. (2008): Theorie des Erzahlens. Göttingen: Vandenhoeck \& Ruprecht. 8. Auflage.

Tokarczuk, Olga (2020a): Czuły narrator. Kraków: Wydawnictwo Literackie.

Tokarczuk, Olga (2020b): Czuły narrator. In: Olga Tokarczuk: Czuły narrator Kraków: Wydawnictwo Literackie, S. 261-289.

Tokarczuk, Olga (2020c): Der liebevolle Erzähler. Vorlesung zur Verleihung des Nobelpreises für Literatur. Mit dem Essay Wie Übersetzer die Welt retten. Aus dem Polnischen von Lisa Palmes. Zürich: Kampa.

Tokarczuk, Olga (2020d): Palec w soli, czyli krótka historia mojego czytania. In: Olga Tokarczuk: Czuty narrator. Kraków: Wydawnictwo Literackie, S. 93-113.

Tokarczuk, Olga (2020e): Wykłady łódzkie. Psychologia narratora. In: Olga Tokarczuk: Czuły narrator. Kraków: Wydawnictwo Literackie. S. 147-179. 
Trzebiński, Jerzy (2002): Narracyjne konstruowanie rzeczywistości. In: Jerzy Trzebiński (Hg.): Narracja jako sposób rozumienia świata. Gdańsk: Gdańskie Wydawnictwo Psychologiczne, S. 15-42.

Warchala, Jacek (1993): Potoczna narracja w dialogu. In: Aleksander Wilkoń/Jacek Warchala (Hg.): Z problemów wspótczesnego jezyka polskiego. Katowice: Wydawnictwo Uniwersytetu Śląskiego, S. 22-32.

Weinrich, Harald (1972): Semantik der Metapher. In: Walter A. Koch (Hg.): Studia Semiotica. Strukturelle Textanalyse. Band 1. Hildesheim/New York: Georg Olms Verlag, S. 268-283.

Wiktorowicz, Józef/Frączek, Agnieszka (2008): Wielki stownik polsko-niemiecki. Großwörterbuch Polnisch-Deutsch. Warszawa: Wydawnictwo Naukowe PWN.

Wilkoń, Aleksander (2002): Spójność i struktura tekstu. Wstep do lingwistyki tekstu. Kraków: Universitas.

Witosz, Bożena (2005): Genologia lingwistyczna. Zarys problematyki. Katowice: Wydawnictwo Uniwersytetu Śląskiego.

Witosz, Bożena (2015): Grundlagen der Textsortenlinguistik. Frankfurt a. M.: Peter Lang, übersetzt von A. Hanus und I. Szwed.

\section{Internetquellen (Zitate und Referenzen von Internetquellen)}

Gołek-Sepetliewa, Dorota (2020): Recepcja twórczości Olgi Tokarczuk w Bułgarii. Przekłady Literatur Stowiańskich T. 10, Nr 2 (2020), S. 47-58, doi 10.31261/PLS.2020.10.02.02.

Hillgruber, Katrin (2019): Die Nobelpreis-Rede von Olga Tokarczuk. Auf der Suche nach der zärtlichen Erzählinstanz. In: https://www.tagesspiegel.de/kultur/die-nobelpreis-rede-von-olga-tokarczukauf-der-suche-nach-der-zaertlichen-erzaehlinstanz/25312238.html (12.12.2019).

https://sprachkompass.ch/theorie/metaphern-erkennen (18.12.2020).

https://www.dwds.de/wb/texten (15.12.2020).

Narodowy Korpus Języka Polskiego Pelctra : http://nkjp.pl/ (20.11.2020).

Nobelpreisrede. Tokarczuks Plädoyer für die Sensibilität. In: https://orf.at/stories/3146807/ (12.12.2020). https://en.pons.com/translate/german-polish/z\%C3\%A4rtlich (10.12.2020).

Seele: https://www.duden.de/rechtschreibung/Seele (15.12.2020).

Tokarczuk, Olga (2019): Czuty narrator. In: https://xiegarnia.pl/aktualnosci/olga-tokarczuk-mowanoblowska/ https://xiegarnia.pl (15.10.2020).

https://www.uibk.ac.at/theol/leseraum/bibel/1kor13.htmlgl (10.12.2020).

Wielki słownik jezyka polskiego. In: https://www.wsjp.pl/ (12.12.2020). 